\title{
Identification of Haptoglobin as an Angiogenic Factor in Sera from Patients with Systemic Vasculitis
}

\author{
Maria C. Cid, Derrick S. Grant, * Gary S. Hoffman, Robert Auerbach, ${ }^{\star}$ Anthony S. Fauci, and Hynda K. Kleinman * \\ Laboratory of Immunoregulation, National Institute of Allergy and Infectious Diseases, and * Laboratory of Developmental Biology, \\ National Institute of Dental Research, National Institutes of Health, Bethesda, Maryland 20892; and \\ ${ }^{\ddagger}$ Laboratory of Developmental Biology, University of Wisconsin, Madison, Wisconsin 53706
}

\begin{abstract}
Angiogenesis is an important process in chronic inflammatory diseases. We observed that sera from patients with systemic vasculitis stimulated angiogenesis in an in vitro model using human umbilical vein endothelial cells cultured on a basement membrane (Matrigel) substrate. After $\mathbf{4 0} \%$ ammonium sulfate precipitation, angiogenic activity remained in the low molecular weight fraction and could be inactivated by heat. SDS-page of serum FPLC fractions exhibiting maximal angiogenic activity demonstrated two prominent species of 45 and $16-20 \mathrm{kD}$ in patients' sera. These bands were much less apparent in sera obtained from control subjects. Amino-terminal sequencing of the $45-\mathrm{kD}$ protein demonstrated that it was haptoglobin. Purified haptoglobin stimulated angiogenesis in a dose-dependent manner. The angiogenic activity of vasculitis patients' sera was partially inhibited by an antihaptoglobin antibody. Furthermore, serum haptoglobin levels in vasculitis patients correlated both with disease and angiogenic activity. Haptoglobin angiogenic activity was confirmed in two in vivo models using an implanted disc and a subcutaneous injection of basement membrane. Stimulation of angiogenesis is a newly recognized biological function of haptoglobin. The increased levels of haptoglobin found in chronic inflammatory conditions may play an important role in tissue repair. In systemic vasculitis, haptoglobin might also compensate for ischemia by promoting development of collateral vessels. (J. Clin. Invest. 1993. 91:977-985.) Key words: vasculitis $\bullet$ inflammatory diseases $\bullet$ angiogenesis $\bullet$ basement membrane $\bullet$ endothelial cell
\end{abstract}

\section{Introduction}

Angiogenesis plays an important role in a variety of physiological and pathological conditions, such as embryonic development, tumor growth, wound healing, and chronic inflammatory diseases (1). The formation of new blood vessels involves dissolution of the underlying basement membrane, followed by cell migration, alignment, proliferation, differentiation into tubular structures, and finally reestablishment of a new basement membrane $(2,3)$. The basement membrane is an important biological mediator of this process and this has been exploited in both in vitro and in vivo assays to assess the angio-

Address correspondence to Hynda K. Kleinman, National Institutes of Health, National Institute of Dental Research, Building 30, Room 407, Bethesda, MD 20892.

Received for publication 7 May 1992 and in revised form 19 October 1992.

The Journal of Clinical Investigation, Inc.

Volume 91, March 1993, 977-985 genic activity of various factors (4-7). Endothelial cells grow as a monolayer on plastic, but when plated on a reconstituted basement membrane (Matrigel), they cease to proliferate and differentiate into a complete network of capillary-like structures within 12-18 h $(8,9)$. To undergo differentiation, endothelial cells require unidentified soluble factors present in serum (10). The Matrigel in vitro model has significant advantages compared with other methods in which only a small percentage of the cells differentiate during a period of time ranging from one to several weeks and in which quantitation is difficult $(11,12)$. Matrigel can also be injected subcutaneously in mice and used as a vehicle to assess angiogenic activity of different compounds $(13,14)$. Subcutaneous injection of Matrigel alone is not associated with focal angiogenesis. However, if fibroblast growth factor-heparin is mixed with Matrigel before injection, ingrowth of vessels is observed within days. The Matrigel plugs can be easily removed by dissection, fixed, stained and the newly formed vessels can be quantitated $(13,14)$. To date, the factors tested in vitro and in vivo Matrigel assays show similar activities to those observed in the chick chorioallantoic membrane (9, 13-15).

Systemic vasculitis includes a heterogeneous group of disorders characterized by inflammation of blood vessels. Examples of major systemic vasculitic syndromes are polyarteritis nodosa, Churg-Strauss syndrome, Wegener's granulomatosis (WG), ${ }^{1}$ giant-cell (temporal) arteritis, and Takayasu's disease $(16,17)$. The inflammatory process often leads to the occlusion or stenosis of the vascular lumen either by thrombus or by both myointimal proliferation and fibrosis, resulting in ischemia or infarction. Histopathologic studies of affected tissues often reveal new reparative vessels (reference 18; Cid, M. C., et al., unpublished observations). The factor(s) that trigger this angiogenic response are not known. We have tested sera from vasculitis patients at various stages of the disease and have found potent angiogenic activity using both in vitro and in vivo angiogenesis models. Haptoglobin, which is not known to have angiogenic properties, was identified as one of the angiogenic substances present in serum.

\section{Methods}

Patient material. Serum samples were obtained from 38 individuals with systemic vasculitis and from 19 healthy blood donors. 28 patients had WG, 6 had biopsy-proven giant-cell (temporal) arteritis, and 4 had Takayasu's disease. 11 WG patients had limited disease, clinically restricted to upper and/or lower airways and 17 had generalized WG

1. Abbreviations used in this paper: FPLC, fast performance liquid chromatography; Hpr, haptoglobin-related protein; HUVEC, human umbilical vein endothelial cells; WG, Wegener's granulomatosis. 
including kidney involvement. When serum samples were obtained, 16 WG patients had active disease, 5 patients had inactive disease with immunosuppressive therapy, and 9 patients were in complete remission without treatment. Two patients were tested twice during active and inactive disease. All temporal and Takayasu's arteritis patients had evidence of active disease.

Endothelial cell culture. Human umbilical vein endothelial cells (HUVEC) from fresh placental cords were isolated by previously described methods (19) and grown until confluence at $37^{\circ} \mathrm{C}$ in $5 \% \mathrm{CO}_{2}$. The growth medium consisted of Medium 199 (Gibco Laboratories, Grand Island, NY) supplemented with $20 \%$ bovine calf serum (Hyclone Laboratories Inc., Logan, UT), $200 \mu \mathrm{g} / \mathrm{ml}$ endothelial cell growth supplement (Collaborative Research, Bedford, MA), $100 \mathrm{U} /$ $\mathrm{ml}$ penicillin-streptomycin, $50 \mu \mathrm{g} / \mathrm{ml}$ gentamicin, $2 \mathrm{mM}$ glutamine (Gibco Laboratories), and $50 \mathrm{U} / \mathrm{ml}$ sodium heparin (Fisher Scientific, Fair Lawn, NJ). Cells used for experiments were from passage 4 to 8 .

In vitro angiogenesis model. The reconstituted basement membrane, Matrigel, was extracted from the murine Engelbreth-HolmSwarm tumor as previously described (20), sterilized with chloroform, and dialyzed against Medium 199. 24-well plates (Costar Corp., Cambridge, MA) were coated with $300 \mu \mathrm{l}$ of Matrigel per well, which was allowed to polymerize at $37^{\circ} \mathrm{C}$ for $30 \mathrm{~min}$. $\operatorname{HUVEC}(30,000$ per well $)$ suspended in Medium 199 were plated on Matrigel and either patients' or controls' sera at a concentration of $5 \%$ in a final volume of $1 \mathrm{ml}$ were added. These conditions of low serum and low cell number are suboptimal for endothelial cell differentiation in this assay $(9,10)$ and were selected so as to detect factors that promote tube formation. After an overnight incubation at $37^{\circ} \mathrm{C}$ in $5 \% \mathrm{CO}_{2}$, the capillary-like structures (tubes) were fixed and stained with Diff-Quik (Baxter Healthcare Corporation, McGaw Park, IL) and the total tube area per well in duplicate wells was measured at $10 \times$ magnification with a computerized digital analyzer (Optomax Inc., Hollis, NH). In subsequent experiments, commercial haptoglobin purified from pooled human plasma (pheno- types 1.1, 2.1, and 2.2; Sigma Chemical Co., St. Louis, MO) was tested in triplicate in the same system at different concentrations ranging from 10 to $100 \mu \mathrm{g} / \mathrm{ml}$. In addition, the purified IgG fraction of either a rabbit anti-human haptoglobin antiserum (Sigma Chemical Company) or nonimmune rabbit serum (Zymed Laboratories Inc., San Francisco, CA ) was added to the culture medium at a 1:20 dilution and tube formation was assessed as described above. Each assay was repeated three times with comparable results.

Purification of the angiogenic factor ( $s$ ) from WG serum. Samples of medium containing $14 \%$ serum from either patients or controls were precipitated with $40 \%$ ammonium sulfate. The supernate and the precipitate were dialyzed against M199 and angiogenic activity was tested at 1:2 dilution in the in vitro Matrigel assay.

$1 \mathrm{ml}$ of the $40 \%$ ammonium sulfate supernate was applied to an FPLC column (Superose-6; Pharmacia LKB Biotechnology Inc., Piscataway, NJ) and eluted with DPBS- Gibco Laboratories). The angiogenic activity of the fractions obtained was tested in the in vitro Matrigel assay in the presence of $2 \%$ BCS. The fractions with highest activity were dialyzed against distilled $\mathrm{H}_{2} \mathrm{O}$, lyophilized, and electrophoresed in 10\% SDS-PAGE under reducing conditions as previously described (21).

Amino acid sequencing. Active fractions from the FPLC column from different patients' serum samples were electrophoresed in SDSPAGE, transferred onto a nylon sheet (Immobilon; Millipore Corporation, Bedford, MA), fixed with $100 \%$ methanol, and stained with freshly prepared $0.1 \%$ Coomassie brilliant blue R-250 (Sigma Chemical Co.) in $50 \%$ methanol $/ 10 \%$ acetic acid. The amino-terminal sequencing of the $45 \mathrm{kD}$ band that appeared in the active fractions was performed directly from Immobilon paper by Matt Williamson (University of California, San Diego).

Haptoglobin serum levels determination. Haptoglobin serum concentration was measured by nephelometry (American Medical Laboratories, Fairfax, VA) in 25 patients with WG ( 10 with active disease, 6
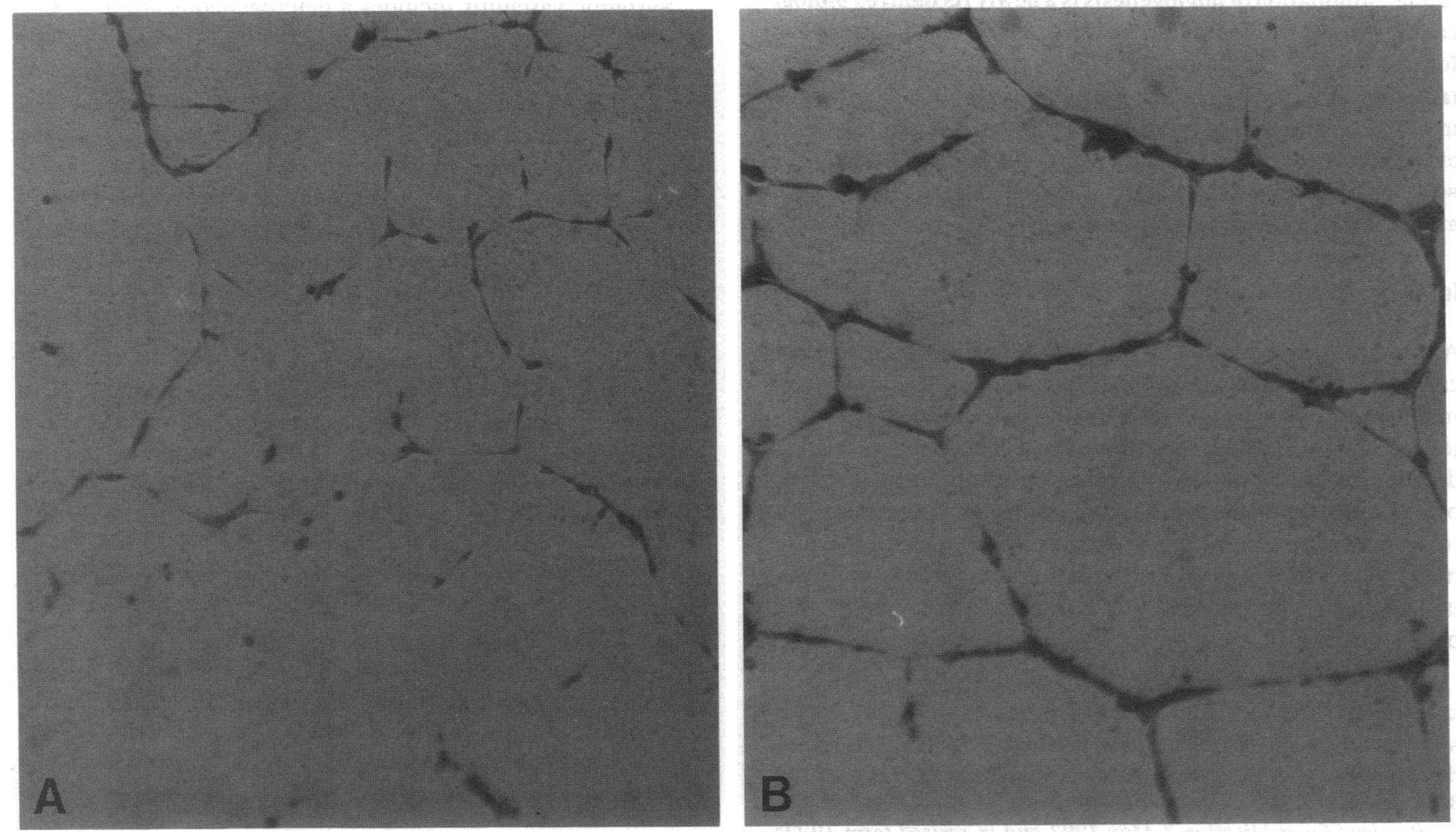

Figure 1. Endothelial cell differentiation on Matrigel. At low cell density and at low serum concentration, sera from systemic vasculitis patients strongly stimulate endothelial cell differentiation into capillary-like structures. $(A)$ Only fragments of unconnected tubes appear with control serum. $(B)$ Complete network induced by WG serum. Tubes are fixed and stained with Diff-Quik. $\times 50$. 
who were asymptomatic and receiving immunosuppressive therapy, and 9 who were untreated and in remission) and 10 healthy controls.

In vivo Matrigel assays. Matrigel (liquid at $4^{\circ} \mathrm{C}$ ) was mixed with various concentrations of commercially obtained (Sigma Chemical Co.) human haptoglobin 1.1 or haptoglobin 2.2 at a final concentration of $0.5,1$, and $2 \mathrm{mg} / \mathrm{ml}$. In some experiments, haptoglobin was first heat inactivated at $100^{\circ} \mathrm{C}$ for $15 \mathrm{~min}$. Matrigel alone or mixed with test haptoglobin $(0.5 \mathrm{ml}$ total volume $)$ was injected subcutaneously into immunodeficient mice (NIH beige nude XID) in duplicate $(13,14)$. After $10 \mathrm{~d}$, the animals were killed, and the Matrigel plugs were removed together with the abstract epidermis and dermis, fixed in $3.7 \%$ formaldehyde, and embedded in paraffin. Histological sections were stained with Masson's trichrome. The vessel area in histological sections of the Matrigel plug was measured using a computerized digital analyzer (Optomax Inc.). A total of 20 fields ( $100 \times$ magnification) were evaluated for each specimen. The experiment was repeated three times.

In vivo disc angiogenesis model. This assay was performed essentially as described by Fajardo et al (22) with minor modifications (23). The central core of a polyvinyl sponge disc was removed and permitted to absorb $40 \mu \mathrm{g}$ of either haptoglobin purified from pooled human plasma containing all three isotypes $(1.1,2.1,2.2)$ (Calbiochem Corp., San Diego, CA), purified haptoglobin 1.1, purified haptoglobin 2.2 (Sigma Chemical Co.) or PBS as a negative control. The loaded sponge core was then coated with ethylene vinyl copolymer (Elvax) to achieve a slow release preparation. The core was reinserted into the sponge disc and the whole disc was covered on its broad surfaces by impermeablized filter paper. Assembled sponges were then inserted subcutaneously into the flanks of adult (athymic) mice and recovered after $14 \mathrm{~d}$ in vivo.

Discs were embedded in paraffin, sectioned tangentially $(20 \mu \mathrm{m})$, and stained with hematoxylin/eosin to permit visualization of the area of the disc penetrated by migrating cells. Discs were analyzed using an image analysis system to determine the percent of disc area penetrated by cells.

\section{Results}

Angiogenic activity of vasculitis patients' sera. At a low (5\%) control serum concentration and low cell density, the formation of capillary-like tubes by HUVEC plated on Matrigel was marginal and many incomplete tubes were formed. In contrast, under the same conditions, sera from patients with systemic vasculitis stimulated tube formation on Matrigel (Fig. 1). Sera from WG patients had greater angiogenic activity than those from patients with either Takayasu's disease or giant-cell (temporal) arteritis (Fig. $2 \mathrm{~A}$ ). Hence, serum samples from WG patients were used in all the subsequent experiments. Serum angiogenesis assays performed during different stages of WG disease activity demonstrated that angiogenic activity persisted longer than clinical signs of active disease, although the angiogenic activity did decline when patients achieved complete remission (Fig. 2 B).

Purification of the angiogenic factor(s) from WG sera. Initial characterization of the angiogenic factor(s) present in WG serum demonstrated that the angiogenic activity remained in the serum supernate after $40 \%$ ammonium sulfate precipitation. The capillary-like network obtained with the serum supernate was complete and indistinguishable from that obtained with whole serum. The angiogenic activity was absent from the $40 \%$ ammonium sulfate pellet and was completely abolished by heating the serum supernate. These data suggested that the activity resided in the low molecular weight protein fraction.

The $40 \%$ ammonium sulfate supernate was chromato-
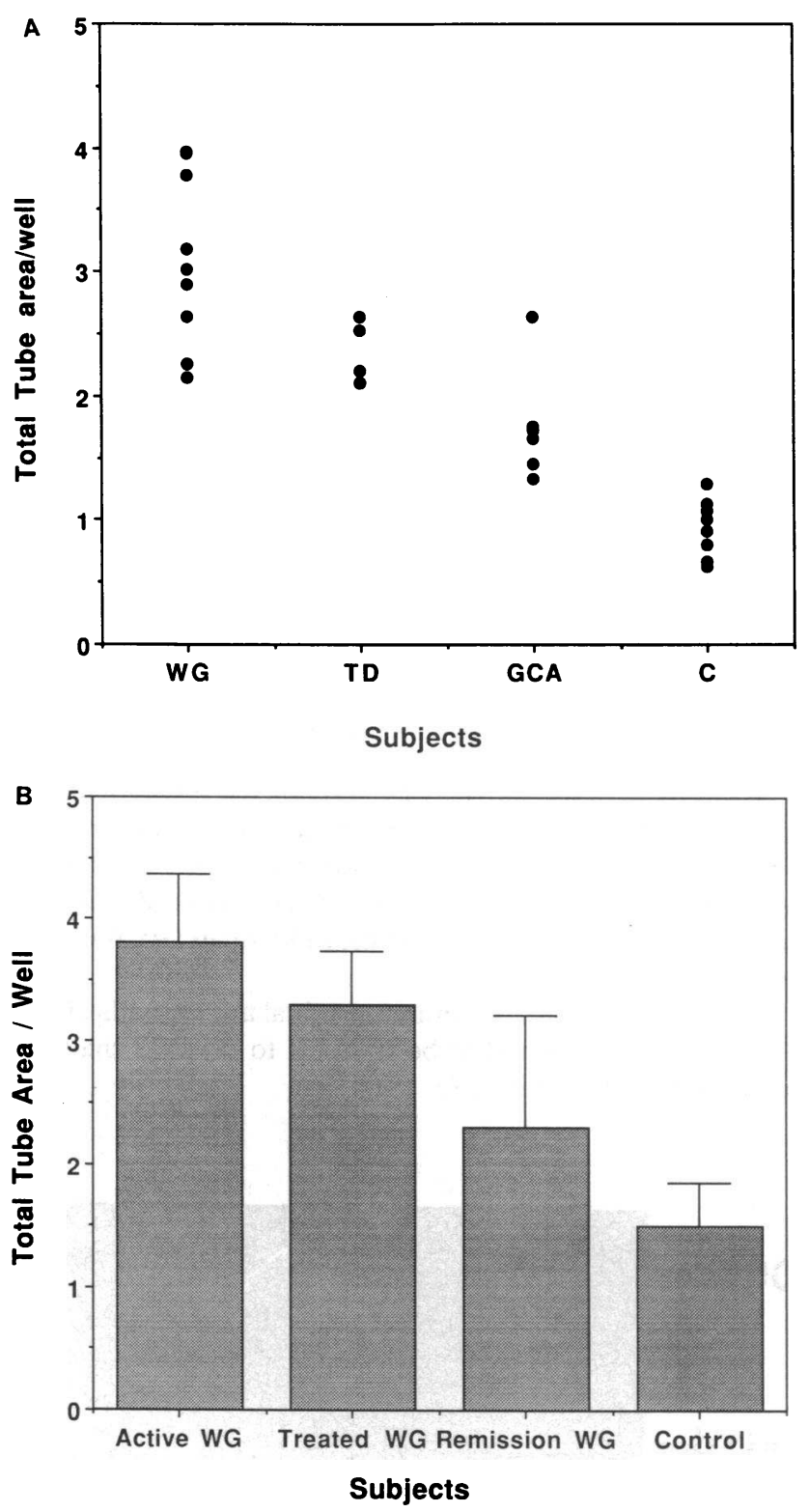

Figure 2. (A) Angiogenic activity in serum samples from different vasculitis patients. Patients include eight with Wegener's granulomatosis $(W G)$, four with Takayasu's disease (TD), six with giant-cell (temporal) arteritis $(G C A)$, and eight controls $(c)$. Each dot represents the average of the tube area per well in duplicate for each sample. Tube area is expressed in $\mathrm{mm}^{2}$. (B) Angiogenic activity of WG patients' sera at different stages of the disease. This experiment includes serum samples from 10 patients with active disease, 4 patients receiving therapy, 4 untreated patients in remission, and 6 controls. Bars represent the mean + SE of the values obtained as described above in each group of individuals.

graphed on Superose 6 by FPLC to purify the factor responsible for the increase in angiogenic activity observed in WG sera. A consistent difference in optical density profiles was observed in nine patients with WG and three normal controls. The WG sera had a larger peak in fractions $35-40$ (Fig. 3 ). When all the fractions were tested in the in vitro Matrigel tube assay, these same fractions were found to contain the maximal angiogenic activity. 


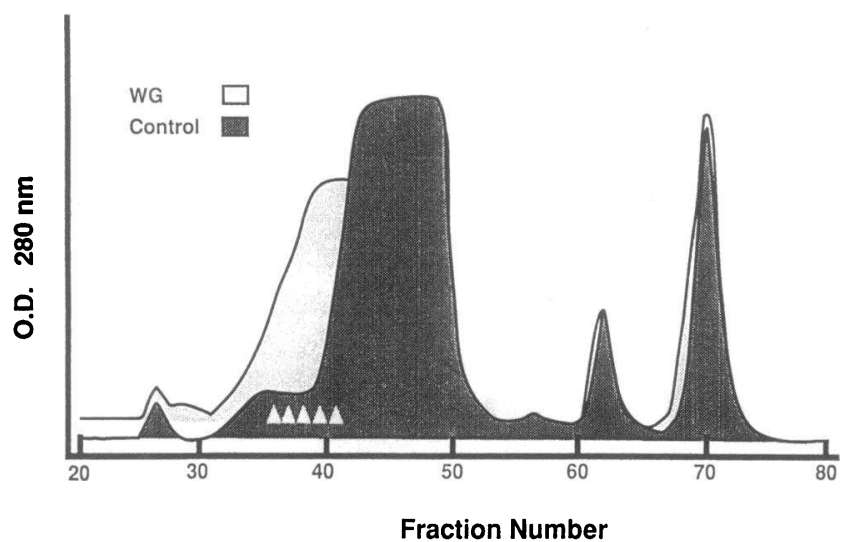

Figure 3. Optical density profile of WG and control serum supernates after $40 \%$ ammonium sulfate precipitation in the first 100 FPLC fractions. WG fractions contained a larger peak in 35-40 fractions, which manifested the highest angiogenic activity.

SDS-PAGE of the active FPLC fractions of WG sera under reducing conditions showed two bands of 45 and $16 \mathrm{kD}$, respectively. These same bands could be detected in fractions from the control sera but their levels were greatly reduced (Fig. 4). This difference was consistent in nine individuals ( six WG and three controls) tested.

The sequence of the 18 amino-terminal amino acids of the $45 \mathrm{kD}$ protein was found to be identical to the beta chain of human haptoglobins (24-27).
Haptoglobin levels were elevated during active disease in WG patients, decreased during treatment and improvement, and were similar to controls during untreated remission (Fig. 5 $A$ ). No differences in haptoglobin concentration were found among WG patients with different patterns of organ involvement. The angiogenic activity tested simultaneously in the in vitro Matrigel assay in 14 WG patients ( 6 active, 3 treated, and 5 in remission) and 5 controls correlated with the corresponding haptoglobin levels (Fig. $5 \mathrm{~B}$ ).

Haptoglobin stimulation of endothelial cell differentiation in vitro. Purified haptoglobin stimulated tube formation in a dose-dependent manner (Fig. 6). Tube-forming activity required the baseline presence of $2 \%$ bovine calf serum, indicating that haptoglobin is necessary but not sufficient to stimulate tube formation. Of the three major haptoglobin phenotypes, haptoglobin 2.2 was most active, the heterozygous 2.1 had intermediate activity, and haptoglobin 1.1 had the least activity (data not shown).

The angiogenic activity of $7 \%$ WG serum was partially inhibited by the IgG fraction of a rabbit antihuman haptoglobin antiserum. Tubes remained well constituted and the inhibitory effect was mainly a decrease in the branching points and in the number of interconnected tubes (Fig. 7). A similar inhibitory effect of the antihaptoglobin antibody was observed on the tube-forming activity promoted by complete medium which contains $20 \%$ bovine calf serum, or on the lesser activity present in 7\% normal human control sera (data not shown). The addition of nonimmune rabbit IgG did not influence tube formation (Fig. 7). These data suggest that one of the active angiogenic compounds present in serum is haptoglobin.
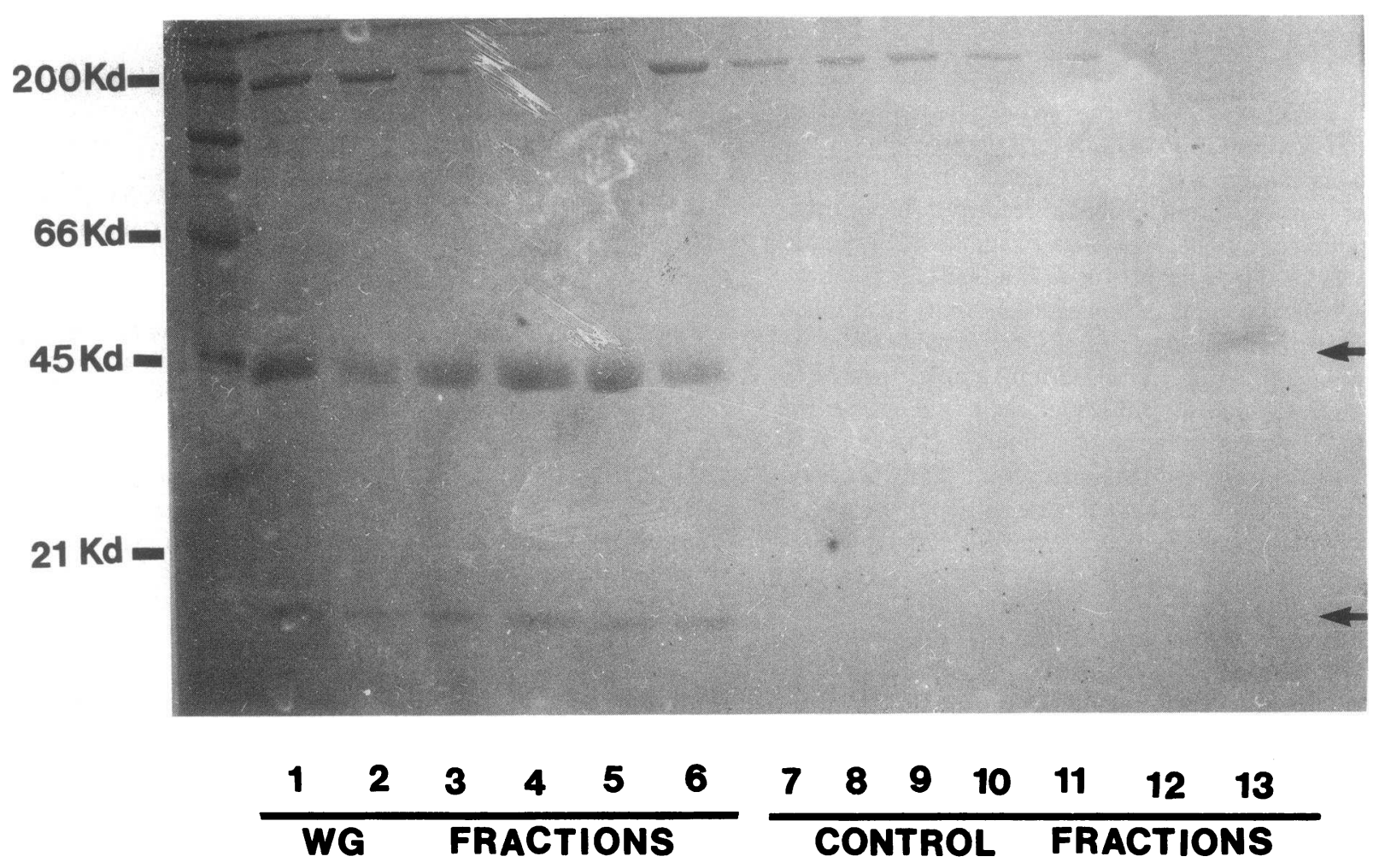

Figure 4. Coomassie blue-stained 10\% SDS-polyacrylamide gel of the FPLC fractions with maximal angiogenic activity shows two bands of $\sim 45$ and $16 \mathrm{kD}$ elevated in the fractions from the WG patient (lanes 1-6) and reduced in the fractions of a normal blood donor (lanes 7-13). 

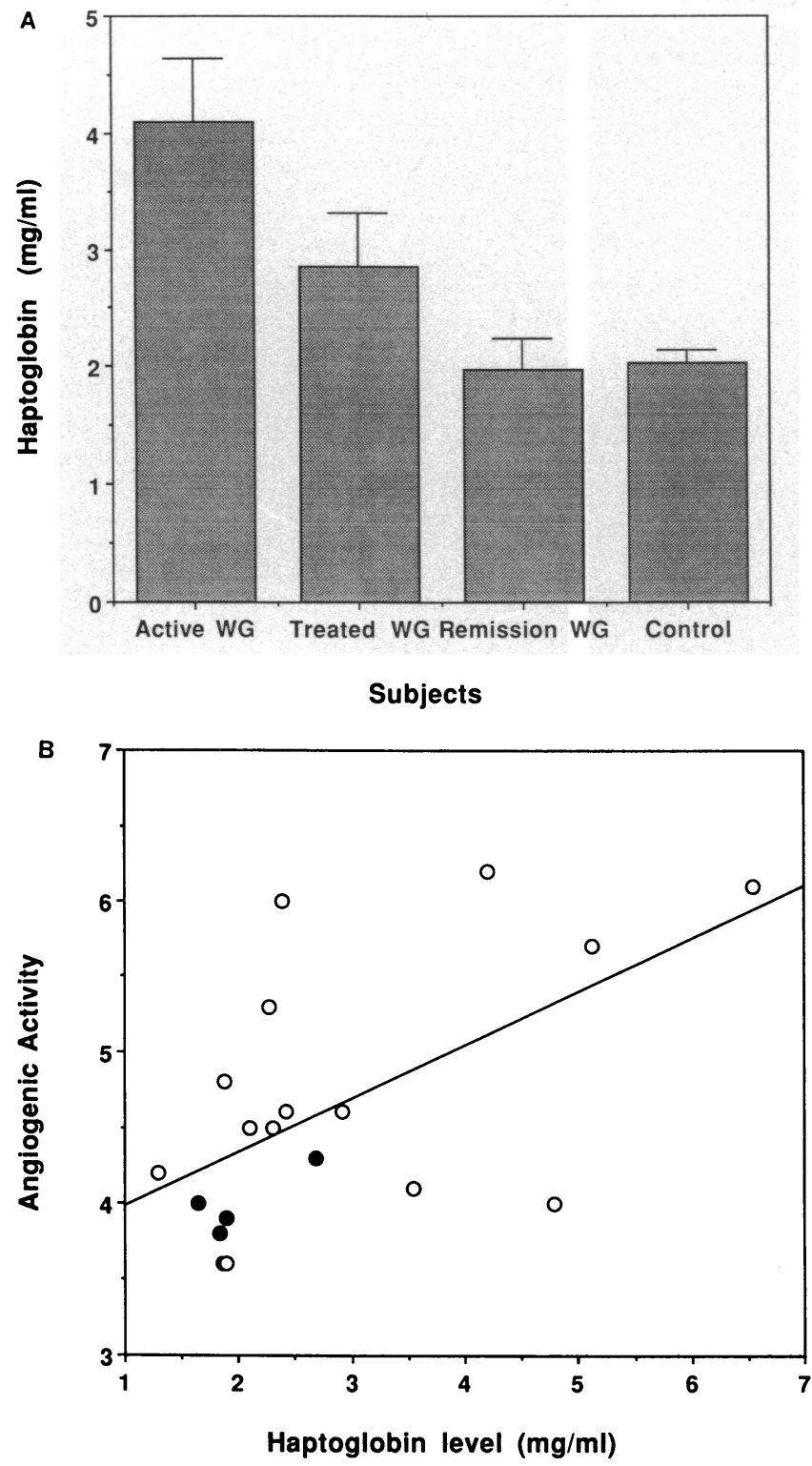

Figure 5. (A) Haptoglobin levels in WG patients at different stages of the disease. Bars represent the mean \pm SE in 10 active patients, 6 asymptomatic patients receiving immunosuppressive treatment, 9 in remission and not requiring therapy, and 10 controls. $(B)$ Significant correlation between haptoglobin levels and the corresponding angiogenic activity of samples from 14 WG patients $(0)$ and 5 controls $(\bullet)(r=0.57, P=0.011)$. WG population includes six patients with active disease, three treated and asymptomatic, and five in remission and untreated.

In vivo angiogenesis models. In vivo, haptoglobin stimulated vascularization of the subcutaneously injected Matrigel plug in a dose-dependent manner and its activity was abrogated by prior heating (Figs. 8 and 9 ). In plugs containing Matrigel alone, only a few cells invaded the plug at the periphery. Haptoglobin-containing plugs differed from those containing Matrigel alone, not only in the density but in the depth that endothelial cells invaded the matrix. In addition, when haptoglobin was present, well- constituted and functional vessels

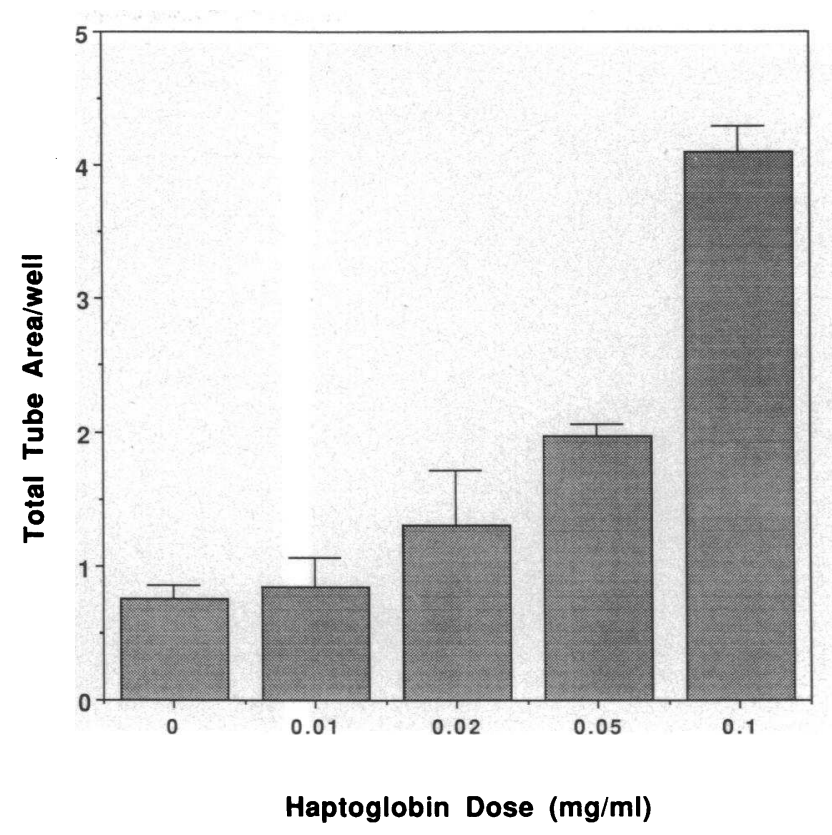

Figure 6. Dose response of the angiogenic activity of purified haptoglobin 2.2 on Matrigel in vitro. Bars represent the mean $\pm S E$ of the total tube area in triplicate wells.

were observed (Fig. 8). The angiogenic effect was apparent at a concentration of $2 \mathrm{mg} / \mathrm{ml}$, which is within the high physiological range. In this in vivo model, haptoglobin 2.2 was much more active than haptoglobin 1.1 , as was noted in the in vitro assay described above.

To exclude any additional effect of Matrigel beside providing extracellular matrix compounds and a physical system to deliver factors to be tested, we confirmed our experiments in another in vivo angiogenesis model: the disc angiogenesis system. In this model, haptoglobin 1.1 induced vessel penetration in $19.8 \pm 4.4 \%$ (mean $\pm \mathrm{SE}$ ) of the disc area; haptoglobin from pooled plasma, $22.9 \pm 2.2 \%$; and haptoglobin $2.2,27 \pm 2.9 \%$. In PBS-treated discs, blood vessels invaded only $15.5 \pm 2.5 \%$ of the disc surface. In the disc angiogenesis model, the angiogenic activity induced by $40 \mu \mathrm{g}$ of haptoglobin 2.2 was equivalent to that achieved with 2-4 ng of basic fibroblast growth factor.

\section{Discussion}

The present study demonstrates that sera from systemic vasculitis patients manifest angiogenic properties. The isolation and purification of serum angiogenic fractions led to the identification of haptoglobin as one of the angiogenic factors. Antihaptoglobin antibodies partially inhibited the angiogenic activity promoted by regular endothelial growth medium, which contains $20 \%$ bovine calf serum, suggesting that haptoglobin may be one of the substances normally present in serum that is required for endothelial cell differentiation. Purified haptoglobin required the presence of $2 \%$ serum to show an angiogenic effect, indicating that it is necessary but not sufficient for angiogenic stimulation in vitro with HUVEC on Matrigel. In vivo, haptoglobin was angiogenic in both the subcutaneous disc and Matrigel implant model systems. 

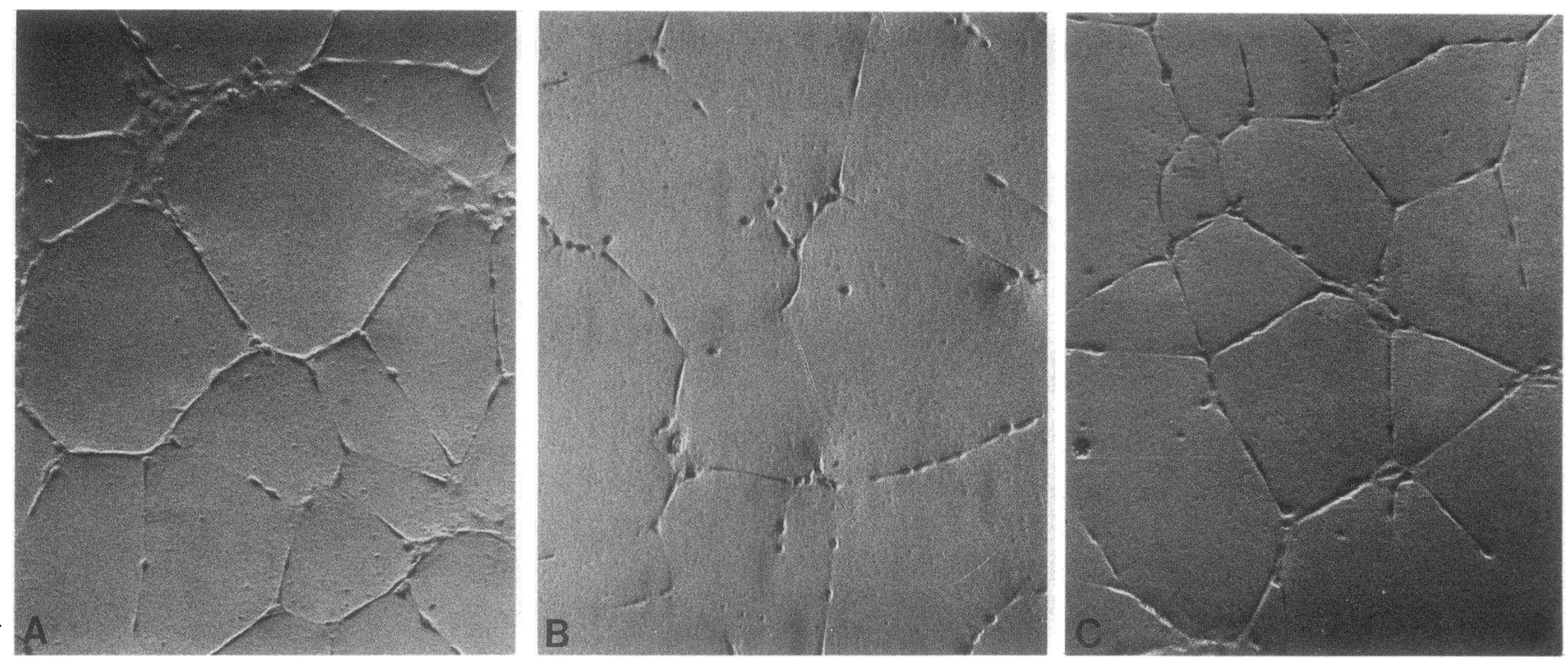

Figure 7. The angiogenic activity of WG serum is partially inhibited by an antihaptoglobin antibody. $(A)$ Tube formation induced by $7 \%$ WG serum. $(B)$ Tube formation promoted by $7 \%$ WG serum after the addition of an antihaptoglobin antibody. $(C)$ Tube formation stimulated by WG serum and a nonimmune rabbit IgG. Hoffman modulation contrast of nonstained tubes. $\times 30$.

Haptoglobin is a plasma alpha $a_{2}$-glycoprotein consisting of an alpha and a beta polypeptide chain of $16-20 \mathrm{kD}$ and $45 \mathrm{kD}$, respectively, linked by disulfide bonds that originate after proteolytic cleavage from a single precursor (24-27). Human haptoglobin has three major allelic forms, haptoglobin 1.1, haptoglobin 2.2 , and the heterozygous 2.1 , which are defined by differences in the alpha chain. The human haptoglobin gene has been cloned and the entire genomic DNA sequenced (28). A haptoglobin-related sequence ( $\mathrm{Hpr}$ ) is located $2.2 \mathrm{~kb}$ downstream of the haptoglobin gene (28). It is unclear if $\mathrm{Hpr}$ is expressed and the function of its theoretical product is still unknown. The haptoglobin gene is expressed in hepatocytes and in several hepatoma cell lines where it is upregulated by several cytokines, particularly IL-6 (29-33).

The best known biological function of haptoglobin is as a carrier for free hemoglobin. The high affinity haptoglobin-hemoglobin complex is taken up by hepatocytes and catabolized $(34,35)$. The adaptative value of this process appears to be prevention of both iron loss and kidney damage during hemolysis. In addition, plasma levels of haptoglobin increase in both acute and chronic inflammatory diseases $(36,37)$. In these situations, it has been considered to be part of a nonspecific response triggered by systemically released cytokines that cause altered hepatic synthesis of major plasma proteins (30-32). The significance of increased levels of haptoglobin in these situations has not been clearly elucidated.

Our studies demonstrate angiogenic activity as a newly recognized function of haptoglobin. Persistent elevations of serum haptoglobin in the setting of inflammation and ischemia may play a role in compensatory angiogenesis. Although angiogenesis is a complex process affected by a variety of factors, we found a significant correlation between serum haptoglobin levels in patients with WG and serum angiogenic activity. Therefore, the angiogenic activity of haptoglobin may be important in chronic inflammatory diseases where it can promote tissue repair. In systemic vasculitis, it may compensate for ischemia by stimulating the development of collateral vessels. The per- sistence of serum angiogenic activity and elevated serum haptoglobin levels beyond the period of clinically active disease in WG further suggests an important reparative role for this protein.

Elevated serum concentrations of haptoglobin also occur in the setting of pregnancy (38), myocardial infarction (39), and cancer $(39,40)$. In a variety of malignancies, haptoglobin concentration correlates with disease progression (40-43). Angiogenesis is an important phenomenon in all of these conditions (44-46). In myocardial infarction, the haptoglobin 2.2 phenotype is associated with a worse prognosis defined by a greater incidence of left ventricular failure and shorter survival (47, 48 ). It has been suggested that the worse prognosis of myocardial infarction in patients carrying the haptoglobin 2.2 phenotype could be explained by a deleterious effect of haptoglobin 2.2 itself. Conversely, haptoglobin-stimulated microangiogenesis may have allowed patients with atherosclerosis to be spared myocardial infarction until more advanced stages of coronary disease (47). Our observation that haptoglobin 2.2 is more angiogenic than other phenotypes supports the latter hypothesis. If the angiogenic activity of haptoglobin is relevant in vivo in these conditions, other unknown factors must regulate and target its effects where required.

The enhanced angiogenic activity present in vasculitis sera compared with controls may be due to quantitative differences in haptoglobin concentration or to the presence of a different haptoglobin with higher angiogenic activity. Immunochemically and functionally different haptoglobins have been described in other pathological conditions. For example, haptoglobin purified from malignant effusions is structurally similar to fetal haptoglobin and has immunosuppressive properties that are more potent than that of adult haptoglobin (42-44). Cancer or fetal haptoglobins and normal adult haptoglobin differ primarily because of postranslational modifications that include variations in glycosylation $(42,43)$. Abnormally glycosylated forms of haptoglobin have also been described in chronic inflammatory diseases such as rheumatoid arthritis 

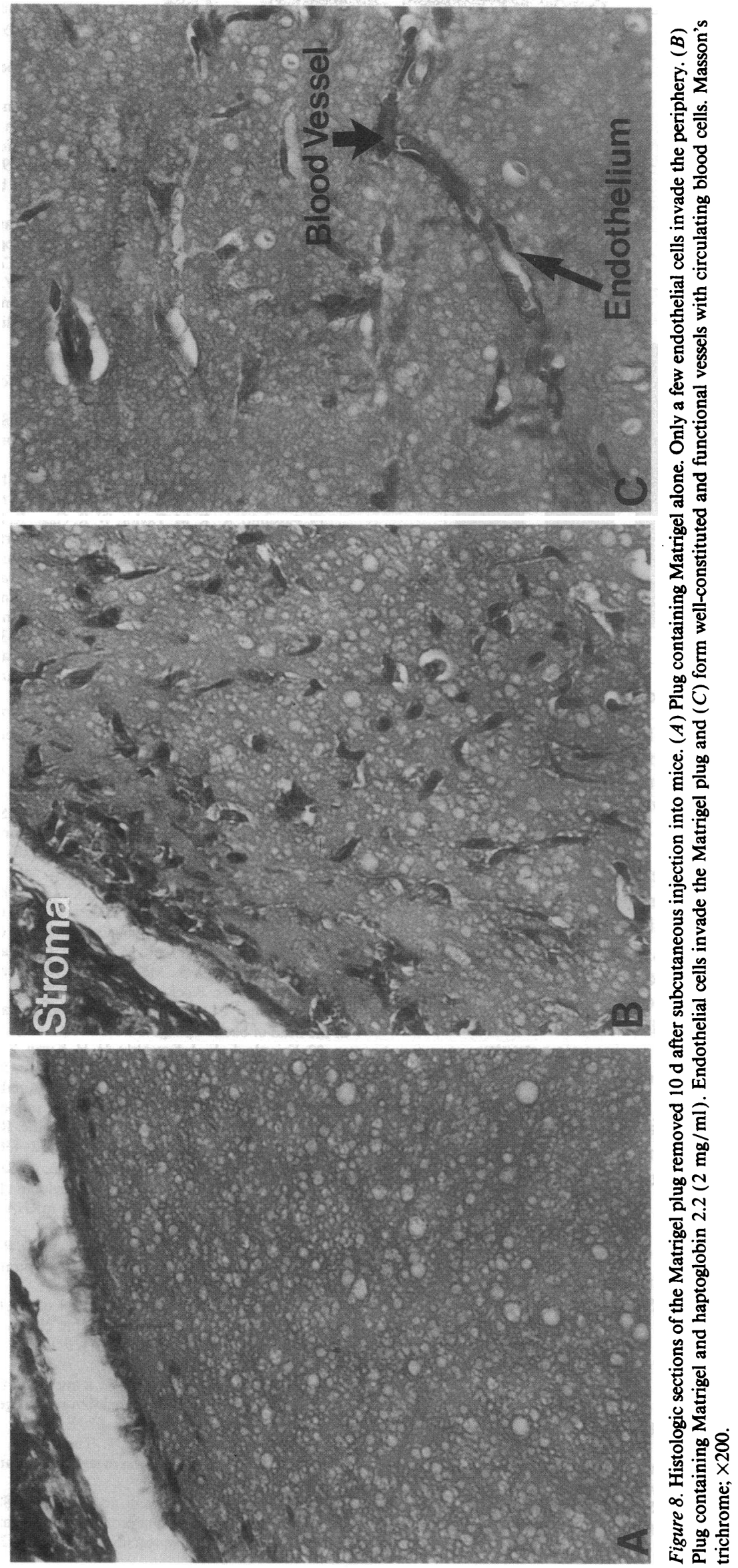


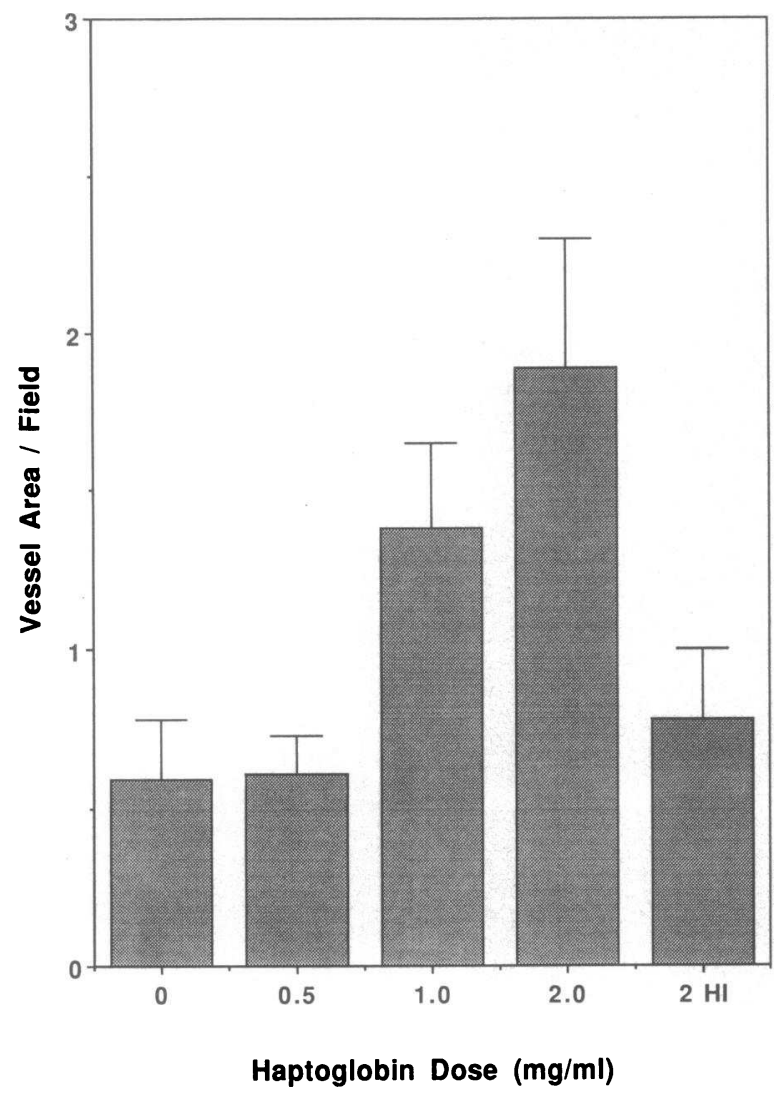

Figure 9. Dose response of purified haptoglobin 2.2 in the in vivo Matrigel angiogenesis assay. Bars represent the mean \pm 1 SE of the vessel area in 20 fields of each specimen. Heat inactivation $(H I)$ strongly reduces haptoglobin activity.

(49). Immunohistochemical detection of haptoglobin with epitopes corresponding to the predicted sequence of the $\mathrm{Hpr}$ product has been recently described in advanced prostatic tumors $(50)$ and in breast cancer, where its presence in initial stages correlates with the occurrence of early metastasis (51). In the present study, we did not determine whether the haptoglobin present in patients' sera has structural differences that could result in an increase in its angiogenic activity.

In summary, our studies demonstrate that haptoglobin stimulates angiogenesis in both in vitro and in vivo models. Haptoglobin elevation in chronic inflammatory and/or ischemic conditions may be important for tissue repair and in promoting growth of collateral vessels.

\section{Acknowledgments}

M. C. Cid was supported by a grant from Fondo de Investigaciones Sanitarias de la Seguridad Social (FISSS 91/5310) and by the Fogarty visiting program.

\section{References}

1. Folkman, J., and M. Klagsbrun. 1987. Angiogenic factors. Science (Wash DC). 235:442-447.

2. Furcht, L. T. 1986. Critical factors controlling angiogenesis: cell products, cell matrix, and growth factors. Lab. Invest. 55:505-509.

3. Liotta, L. A., P. S. Steeg, and W. G. Stetler-Stevenson. 1991. Cancer metastasis and angiogenesis: an imbalance of positive and negative regulation. Cell. $64: 327-336$.
4. Madri, J. A., S. K. Williams, T. Wyatt, and C. Mezzio. 1983. Capillary endothelial cell cultures: phenotype modulation by matrix components. J. Cell. Biol. 97:153-166.

5. Ingber, D. E., and J. Folkman. 1989. How does extracellular matrix control capillary morphogenesis? Cell. 58:803-805.

6. Ingber, D. E., and J. Folkman. 1989. Mechanochemical switching between growth and differentiation during fibroblast growth factor-stimulated angiogenesis in vitro: role of extracellular matrix. J. Cell. Biol. 109:317-330.

7. Form, D. M., B. M. Pratt, and J. A. Madri. 1986. Endothelial cell proliferation during angiogenesis. In vitro modulation by basement membrane components. Lab. Invest. 55:521-530.

8. Kubota, Y., H. K. Kleinman, G. R. Martin, and T. J. Lawley. 1988. Role of laminin and basement membrane in the morphological differentiation of human endothelial cells into capillary-like structures. J. Cell. Biol. 107:1589-1598.

9. Grant, D. S., K. I. Tashiro, B. Segui-Real, Y. Yamada, G. R. Martin, and H. K. Kleinman. 1989. Two different laminin domains mediate the differentiation of human endothelial cells into capillary-like structures in vitro. Cell. 58:933-943.

10. Kinsella, J. L., D. S. Grant, B. S. Weeks, and H. K. Kleinman. 1992. Protein kinase $\mathrm{C}$ regulates endothelial cell tube formation on basement membrane Matrigel. Exp. Cell. Res. 199:56-62.

11. Montesano, R., L. Orci, and P. Vassali. 1983. In vitro rapid organization of endothelial cells into capillary-like networks is promoted by collagen matrices. J. Cell. Biol. 97:1648-1652.

12. Madri, J. A., and B. M. Pratt. 1986. Endothelial cell-matrix interactions: in vitro models of angiogenesis. J. Histochem. Cytochem. 34:85-91.

13. Passaniti, A., R. M. Taylor, W. R. Gage, R. Pilli, Y. Guo, P. V. Long, J. A. Haney, R. R. Pauley, D. S. Grant, and G. R. Martin. 1992. A new quantitative method for assessing angiogenesis and anti-angiogenic agents using reconstituted basement membrane, heparin and FGF. Lab. Invest. 67:519-528.

14. Kibbey, M. C., D. S. Grant, R. Auerbach, and H. K. Kleinman. 1992. The SIKVAV site of laminin promotes angiogenesis and tumor growth in an in vivo Matrigel model. J. Natl. Cancer Inst. 84:1633-1638.

15. Sakamoto, N., M. Iwahana, N. G. Tanaka, and Y. Osada. 1991. Inhibition of angiogenesis and tumor growth by a synthetic laminin peptide, CDPGYIGSR$\mathrm{NH}_{2}$. Cancer Res. 51:903-906.

16. Fauci, A. S., B. F. Haynes, and P. Katz. 1978. The spectrum of vasculitis. Clinical, pathologic, immunologic and therapeutic considerations. Ann. Intern. Med. 89:660-676.

17. Cupps, T. R., and A. S. Fauci. 1981. The Vasculitides. W. B. Saunders, Philadelphia.

18. Olsson, A., P. Elling, and H. Elling. 1990. Serological and immunohistochemical determination of von Willebrand factor antigen in serum and biopsy specimens from patients with arteritis temporalis and polymyalgia rheumatica. Clin. Exp. Rheum. 8:55-58.

19. Jaffe, E. A., R. L. Nachman, C. G. Becker, and C. R. Minick. 1973. Culture of human endothelial cells derived from umbilical veins. Identification by morphological and immunological criteria. J. Clin. Invest. 52:2745-2756.

20. Kleinman, H. K., M. L. McGarvey, J. R. Hassell, V. L. Star, F. B. Cannon, G. W. Laurie, and G. R. Martin. 1986. Basement membrane complexes with biological activities. Biochemistry. 25:312-318.

21. Laemmli, U. K. 1970. Cleavage of structural proteins during the assembly of the head of bacteriophage T4. Nature (Lond.). 227:680-685.

22. Fajardo, L. F., J. Kowalski, H. H. Kwan, S. D. Prionas, and A. C. Allison. 1988. The disc angiogenesis system. Lab. Invest. 58:718-724.

23. Polakowski, I. J., K. Lewis, and R. Auerbach. 1992. A protein RNase inhibitor (RNasin) expresses anti-angiogenic properties in mice. In Angiogenesis, Key Principles, Science, Technology, Medicine. R. Steiner, P. B. Weisz, and R. Langer, editors. Birkhauser Verlag, Basel. 432-436.

25. Raugei, G., G. Bensi, V. Romano, C. Santoro, F. Constanzo, and R. Cortese. 1983. Sequence of human haptoglobin cDNA: evidence that the alpha and beta subunits are coded by the same mRNA. Nucleic Acids Res. 11:58115819.

26. Yang, F., J. L. Brune, W. D. Baldwin, D. R. Barnett, and B. H. Bowman. 1983. Identification and characterization of human haptoglobin cDNA. Proc. Natl. Acad. Sci. USA. 80:5875-5879.

27. vander Straten, A., A. Herzog, P. Jacobs, T. Cabezon, and A. Bollen. 1983. Molecular cloning of human haptoglobin cDNA: evidence for a single mRNA coding for alpha ${ }^{2}$ and beta chains. EMBO (Eur. Mol. Biol. Organ.) J. 2:10031007.

28. Maeda, N. 1985. Nucleotide sequence of the haptoglobin and haptoglobin-related gene pair. The haptoglobin-related gene contains a retrovirus-like element. J. Biol. Chem. 260:6698-6709.

29. Milland, J., A. Tsykin, T. Thomas, A. R. Aldred, T. Cole, and G. Schreiber. 1990. Gene expression in regenerating and acute-phase rat liver. Am.J. Physiol. 259:G340-G347.

30. Iwasa, F., R. A. Galbraith, and S. Sassa. 1988. Effects of dimethyl sulphoxide on the synthesis of plasma proteins in the human hepatoma HepG2. Induction of an acute-phase-like reaction. Biochem. J. 253:927-930. 
31. Darlington, G. J., D. R. Wilson, and L. B. Lachman. 1986. Monocyteconditioned medium, interleukin-1, and tumor necrosis factor stimulate the acute phase response in human hepatoma cells in vitro. J. Cell. Biol. 103:787793.

32. Castell, J. V., M. J. Gomez-Lechon, M. David, T. Andus, T. Geiger, R. Trullenque, R. Fabra, and P. C. Heinrich. 1989. Interleukin-6 is the major regulator of acute phase protein synthesis in adult human hepatocytes. FEBS (Fed. Eur. Biochem. Soc.) Lett. 242:237-239.

33. Oliviero, S., and R. Cortese. 1989. The human haptoglobin gene promoter: interleukin-6-responsive elements interact with a DNA-binding protein induced by interleukin-6. EMBO (Eur. Mol. Biol. Organ.) J. 8:1145-1151.

34. Kino, K., H. Tsunoo, Y. Higa, and H. Nakajima. 1982. Kinetic aspects of hemoglobin-haptoglobin-receptor interaction in rat liver plasma membranes, isolated liver cells, and liver cells in primary culture. J. Biol. Chem. 257:48284833.

35. Kino, K., H. Tsunoo, Y. Higa, M. Takami, H. Hamaguchi, and H. Nakajima. 1980. Hemoglobin-haptoglobin receptor in rat liver plasma membrane. $J$. Biol. Chem. 255:9618-9620.

36. Kushner, I. 1982. The phenomenon of the acute phase response. Ann. $N Y$ Acad. Sci. 389:39-48.

37. Hansen, J. E. S., J. Iversen, A. Lihme, and T. C. Bog-Hansen. 1987. Acute phase reaction, heterogeneity and microheterogeneity of serum proteins as nonspecific tumor markers in lung cancer. Cancer. 60:1630-1635.

38. Kuhajda, F. P., A. I. Katumuluwa, and G. R. Pasternak. 1989. Expression of haptoglobin-related protein and its potential role as a tumor antigen. Proc Natl. Acad. Sci. USA. 86:1188-1192.

39. Agostoni, A., C. Vergani, R. Stabilini, B. Marasini, R. Arcidiacono, A Sbaff, and P. C. Binaghi. 1970. Immunochemical quantitation of acute phase proteins in myocardial infarction. Am. Heart J. 80:313-318.

40. Mueller, W. K., R. Handschumacher, and N. E. Wade. 1971. Serum haptoglobin in patients with ovarian malignancies. Obstet. Gynecol. 38:427-435.
41. Snyder, S., and Ashwell, G. 1971. Quantitation of specific serum glycoproteins in malignancy. Clin. Chim. Acta. 34:449-455.

42. Oh, S. K., D. L. Very, J. Walker, S. Raam, and S. T. Ju. 1987. An analogy jetween fetal haptoglobin and a potent immunosuppressant in cancer. Cancer Res. 47:5120-5126.

43. Oh, S. K., S. H. Kim, and J. E. Walker. 1990. Interference with immune response at the level of generating effector cells by tumor-associated haptoglobin. J. Natl. Cancer Inst. 82:934-940.

44. Folkman, J., K. Watson, D. Ingber, and D. Hanahan. 1989. Induction of angiogenesis during the transition from hyperplasia to neoplasia. Nature (Lond.). 339:58-61.

45. Folkman, J. 1990. What is the evidence that tumors are angiogenesis dependent? J. Natl. Cancer Inst. 82:4-6.

46. Eidner, N., J. P. Semple, W. R. Welch, and J. Folkman. 1991. Tumor angiogenesis and metastasis. Correlation in invasive breast carcinoma. $N$. Engl. J. Med. 324:1-8

47. Chapelle, J. P., A. Albert, J. P. Smeets, C. Heusghem, and H. E. Kulbertus 1982. Effect of the haptoglobin phenotype on the size of a myocardial infarct. $N$. Engl. J. Med. 307:457-463.

48. Panter, S. S., R. P. Bynigton, L. J. England, B. Schnasse, E. Rubin, M Petracek, S. R. Sundby, P. E. Hallaway, and B. E. Hedlund. 1991. Recovery from myocardial infarction is related to haptoglobin genotype. Clin. Res. 39:347A.

49. Thompson, S., R. Stappenbeck, and G. A. Turner. 1989. A multiwel lectin-binding assay using lotus tetranolobus for measuring different glycosylated forms of haptoglobin. Clin. Chim. Acta. 180:227-284.

50. Shurbaji, M. S., T. S. Thurmond, G. R. Pasternack, and F. P. Kuhajda. 1991. Expression of haptoglobin related protein ( $\mathrm{Hpr}$ ) epitopes by prostate carcinoma: a potential prognostic indicator. Lab. Invest. 64:52A

51. Kuhajda, F. P., S. Piantadosi, and G. R. Pasternak. 1989. Haptoglobin-related protein $(\mathrm{Hpr})$ epitopes in breast cancer as a predictor of recurrence of the disease. N. Engl. J. Med. 321:636-641. 